\title{
Integrated sustainable urban design: neighbourhood design proceeded by sustainable urban morphology emergence
}

\author{
S. Vahabzadeh Manesh ${ }^{1}$, M. Tadi ${ }^{2} \&$ F. Zanni ${ }^{1}$ \\ ${ }^{1}$ Dipartimento di Architettura e Pianificazione (DiAP), \\ Politecnico di Milano, Italy \\ ${ }^{2}$ Dipartimento di Scienza e Tecnologie dell'Ambiente Costruito (BEST), \\ Politecnico di Milano, Italy
}

\begin{abstract}
More than three-fourths of the world's population live and work in cities, where up to $80 \%$ of all available energy is consumed and over half of greenhouse gas emissions are created. In this complex condition, sustainable urban development could deal with both urban growth demands as well as environmental concerns. Regarding awareness of the population distribution as it affects energy consumption, this paper sketches out the relationships between urban morphology and energy consumption, providing some new basic design principles to re-shape urban assessment, as well as to design new sustainable neighborhoods as an integrated part of the city.

Our research applies a specific as well as an innovative approach based on a holistic methodology with the consideration of the city as a complex adaptive system with the ability to learn from past encountered conditions. Hence, considering that the energy consumption of the city is not the sum of all its buildings' consumption, and the final emergence of the system is completely different than the individual element's performance, is the main premise of the research. Accordingly, the paper illustrates a simulative method, based on mentioned methodology of complexity, for one whom deals with the sustainable neighborhood designs and urban transformations. The research is not suggesting a unique solution for different contexts, but rather investigating how to control the urban transformation process to achieve a sustainable form.

Keywords: urban transformation, sustainable form, complex adaptive system, modification, integration.
\end{abstract}




\section{Introduction}

Due to the unprecedented population growth rate in the last century, the urban population has taken over the rural inhabitant number for the very first time in history [1]. Rapid urban growth was the first response of cities to fulfil the new population's upward demands. This rapid urban growth accommodated the new population; nevertheless, it also caused further concomitant problems particularly in urban peripheries, where sprawled settlement has emerged. Indeed, the urban transformation, instead of urban growth, would have been the proper remedy to those demands.

Population growth, in one hand, and environmental concerns such as green house gas emissions, in the other, have arisen new demands, to which the city has to respond; moreover, economic crisis together with the daily increment in the cost of energy have given another dimension to these complex problems to which the city must cope. Building affiliation with energy consumption seems to have major role in the urban energy balance of the city; nevertheless, and despite this general belief, the research presented here is based on the main players of energy balance within the city scenario, which is urban morphology and its form. The significance of urban transformation within the context of sustainable urban morphology can deal with diverse problems, i.e. urban growth, environmental and economic problems, simultaneously.

Much research has been done on the urban form correlation with energy consumption of the city; however, the discordant results have left the planners and designers in utter confusion. Hence, this paper tries to explain why prior research has been concluded with irreconcilable results; moreover, unlike prior research, this paper illustrates a simulative design method, based on holistic complexity analysis, with means to clarify how the urban transformation can be performed in order to achieve a sustainable urban form.

\subsection{Simulation versus simplification}

In the holistic approach, persuaded in this paper, the city has been considered as a single entity, the complex adaptive system (CAS) in particular. Mahaffy et al. [2] believes that the city is an energy-using complex system in its own right, with its own varying levels of efficiency and not just a mere aggregation of disconnected energy consumers.

The complex adaptive system is a specific type of complex system which incorporates the cities adaptation capacity and the final performance of the system emerges from every single components performance together. In this approach, [3], urban morphology emerges through continuous modification of existing urban elements, as an adaptive response to the internal and external imposed constraints, over time. In other words, a CAS undergoes the continuous modification process which concludes with the transformation or evolution of the system. According to Sergio Crotti, "urban form does not comprehend the urban shape statically, as a pure analysis of the status quo, but as a form of dynamic changes of urban system". 
The complex adaptive system is comprised of heterogeneous elements, linked together either directly or indirectly, and the final system performance emerges from all of the elements as a whole. Brownlee [5] states that, "Macroscopic patterns emerge from the dynamic and nonlinear interactions of the systems lowlevel (microscopic) adaptive agents. The emergent patterns are more than the sum of their parts, thus the traditional reductionist methodology fails to describe how the macroscopic patterns emerge". Wotton [6] believes that, the complex behaviour of the system and different elements involvement have made the final emergence of the system too complex to be foreseen. In other words, "Due to variation of agents and differential actions and reactions, the system performance is difficult to be anticipated".

Studying a complex system, through simplification methodology, is not the proper approach if more accurate results are to be obtained. There are many valuable researches, carried out via simplification methodology, which help us to understand better the urban energy balance issue; however, simplifying the problem, by neglecting the involved key factor parameters, has led the researches to discordant results. In this regard, Zanni [4] stated that now a days the complexity and heterogeneity of urban materials are too fragmented to be analysed and transformed by an urban deductive logic. For instance, some research results, derived through simplification methodology, illustrate the direct relation between electrical energy consumption of the city and its density, whilst others, [7], show the inverse relation between density and heat loss. Now the arisen question is which one of these to be given privilege in the total energy balance of the city.

The reason behind these irreconcilable results is embedded in the complex behaviour of the CAS; as referred above, the final emergence of the system is difficult to be anticipated.

Making note of a pervious sentiment, through the dynamic and nonlinear interactions of the lower level agents within a system, an emergent pattern can form through the sum of these agents, yet not always depict the accurate description of the final or macroscopic pattern. Hence, simulation is far preferable to simplification of the complex adaptive system in the researches.

\subsection{Members and subsystem}

As mentioned, the CAS is comprised of different members, connected linearly and nonlinearly; the performances of the members, which are categorized in a defined subsystem, either directly rely each other or share the same function in the system. Every CAS consists of many subsystems, whilst each subsystem could be a CAS in and of itself. For instance, the immune system and nervous system are two complex adaptive systems while they are also considered as subsystems in the complex adaptive human body system. These members and subsystems adapt and modify themselves in order to respond to the newly imposed constraints and circumstances.

The CAS experiences two different types of constraints, internal and external forces. Adaptive reactions are the CAS response to these constraint forces. In other words, the CAS has to adapt its reactions due to changing constraints in 
time. This adaptation occurs within or on members of a subsystem, hereafter known as Horizontal Adaptation, and between the different subsystems, hereafter termed Vertical Adaptation.

\subsection{Horizontal adaptation and horizontal optimization (modification)}

As mentioned, the adaptation of existing members in a subsystem, horizontal adaptation, as a response to the newly imposed conditions and constraints, changes the subsystem's performance, which will be the cause of the entire system transformation, over time. Alteration of a bus line of the city to improve the transportation network efficiency is a horizontal adaptation, whilst the transportation network is considered a subsystem and a bus line is a member of it, for instance.

Although the adaptations could respond to the new parochial demands; nevertheless, in some circumstances, the response would not necessarily improve the entire system's performance. Accordingly, Horizontal Optimization could be introduced as specific type of Horizontal adaptation via modification of the existing elements, when the member's adaptations improve both their own subsystem as well as the entire system's performance. The massive construction wave after World War II, was solely an adaptive immediate and temporal answer to the newly risen urge to find dwelling. The mentioned response accommodated the people in short time; however, in many cases, it was the main cause of subsequent problems in peripheries of the historical cities, for instance, which persist until today. Thus, propelling the adaptation process of the complex system, into a right direction, is the main role of designers and planners.

\subsection{Vertical adaptation and vertical optimization (integration)}

Likewise to horizontal optimization, the vertical optimization is a specific kind of adaptation, where the members adapt themselves to optimize the performance of the entire complex system. However unlike the horizontal one which occurs inside each subsystem individually, the vertical optimization takes place between different subsystems. In other words, the subsystems interact symbiotically in order to improve their own performances, and thereby improve the entire system's performance level. The transportation subsystem improvement, which could be cited as an example, ameliorates other involved subsystems in the city, such as the economical subsystem, and vice versa. In other words, vertical optimization is the collaborative superimposition of two or more subsystems, in a way that the adaptation and transformation of each one improves the other ones performances.

One can improve the performances of the entire complex system, utilizing the adaptive behaviours of the complex adaptive system, both horizontal and vertical, by turning the adaptations into optimizations; hereafter, the horizontal optimization is termed Modification, while the vertical optimization is known as Integration. The entire complex system will be transformed by the mentioned symbiotic adaptive behaviours between the elements and subsystems, modification and integration, over time. By boosting the performance of one 
subsystem, through the assistance of the transformation of another subsystem, creates a collaborative relation which ultimately leads to transformation of the complex system in an optimal way. These adaptive features are the key elements of the CAS simulation method utilized in this paper, as illustrated in table 1.

Table 1: $\quad$ Key elements of the CAS simulation method.

\begin{tabular}{|c|c|c|c|}
\hline \multicolumn{2}{|c|}{ Adaptation of existing constituents } & & Transformation of \\
\cline { 1 - 2 } $\begin{array}{c}\text { Modification of } \\
\text { elements in single layer }\end{array}$ & $\begin{array}{c}\text { Integration of } \\
\text { layers }\end{array}$ & & $\begin{array}{c}\text { Time } \\
\text { Complex System }\end{array}$ \\
\hline
\end{tabular}

The following explicates a proposed design method to achieve sustainable neighbourhood form and furthermore sustainable urban form in a larger scale, via complex adaptive system methodology. Unlike other researches which are based on simplification rather than a simulation method, how to simulate the system with the inclusion of its complexity through the holistic approach is the following subject under investigation.

\section{Methodology}

\subsection{Simulative process}

The CAS simulation approach is comprised of three main steps; studying the complex system and its component is the first main step, which is called the Analysis Phase. Selecting the right involved subsystems, which are in the scope of the study's interest, is the key element of this step. Since the study main concern is devoted to the urban morphology and energy consumption of the city, the proper involved subsystems which affect the urban form as well as energy consumption of the city have to be selected. The apt choice of involved subsystems, amongst the numerous subsystems in a complex system, plays the significant role in the final result of complexity simulation. In the selecting process, either neglecting some of the involved layers or a misguided choice of them would cause simplification of the problem and inaccurate results.

The second step is the Hypothesis Phase. In order to optimize the final system performance through transformation of the complex adaptive system, one should improve the involved subsystem performances by vertical and horizontal optimization via modification of the elements and integration of the subsystems. To reiterate, if one imagines each subsystem as a layer, then the complex system would be comprised of different superimposed layers. Folding and superimposing the selected layers collaboratively, in a way in which the transformation of each layer enhances the other one's performance, is the key factor of the main system transformation. The system would be optimized, via transformation, when all of the superimposed layers meet each other and integrate together simultaneously and collaboratively. With the assistance of symbiotic superimposition of the urban layers, urban integration so to speak, one 
can elucidate a way to propel the urban transformation to a decent expected form. Similarly, in a horizontal adaptation within each subsystem's members, collaborative modification of existing elements optimizes each other's performances.

The Appraisal Phase, or the third step, not only monitors the simulative model, but also evaluates the transformed system's performances. In order to carry out this assessment, some indicative tools are required. Thanks to the help of these indicators, one can compare the characteristic performance of the system, prior and after the transformation process. With the help of the indicators, not only the complex system transformation could be guided to the right direction, but also the result of transformation process could be evaluated. Due to the involvement of the different elements and subsystems in a complex adaptive system, [8], the number of indicators, corresponded to the system, is enormous. The apt indicators would illustrate an image of the system's performances, in order to evaluate the final performances of the system, with respect to the initial intentions.

After studying the city and its main constituent subsystems, the principal investigated subsystems in this paper, which affect the urban morphology as well as total energy balance of the city, have been selected as follows:

- Urban volume (built-up mass layer);

- Urban voids (open spaces, streets and etc.);

- Functional layer ( land use layer);

- Transportation layer.

\subsubsection{Urban volume layer}

Urban mass volume, or the built-up layer, is one of the layers of involved subsystems under investigation. As mentioned, urban transformation, in the holistic adaptive methodology, develops in two different phases, modification of each layer members and integration of different layers together. In this methodology, urban mass is considered as a single porous entity comprised of different elements

In horizontal adaptation, every single modification of an existing building or even constructing a new building, not only has to be concluded with the form which optimises the energy performances of its own, but also improves the other existing building's energy performances, fig.1. Each individual building form not only enhances the solar gaining of itself, but also optimizes the other building solar gaining as well, for instances. The modification of every element in this layer, alike the other involved layers, occur with the urban contextual consideration. Needless to say, the region climatic data should be considered; for instance, of the climate control for improving solar exposure is important for some locations, such as cold regions, whilst the solar gaining prevention, such as hot middle-eastern cities, is of high priority in other places, for instances.

In the vertical adaptation, the integration of this layer, volume layer, with the other layers, transportation and functional layer for instance, is another phase of the complex transformation process. The urban density and its volume 


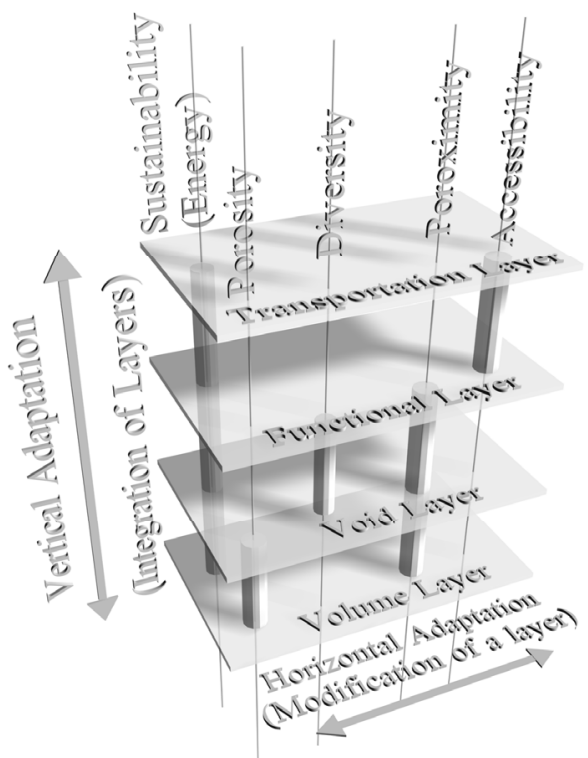

Figure 1: Horizontal and vertical adaptation of the four main principal layers.

collaborate with the transportation layer in order to maximize the efficiency of public and private transportation.

\subsubsection{Urban void layer}

The volume layer clearly defines the presence of this principle layer, the urban void layer; whereby the integration of these two layers, urban volume and void, conveys the physical meaning of the city. Indeed, one can imagine the city as a solid porous volume, sponge like, with various sizes of holes linked by linear porosities.

The significance of the volume and void configuration in the city, spreads in all aspects of the urban design, even from urban space quality to energy aspect of the city. With regards to the field of interest in this paper, namely the cities' energy aspect, the proper configuration of the volume and void, porosity, could reduce the total energy consumption of the city considerably, fig. 1. Solar gaining, shading and natural ventilation and heat loss could be optimized solely by the volume and void integration. There are many historical cities around the worlds, particularly in Asia and Europe, that could be cited as the low energy consumption cities, [9], thanks to the complex integration of closed and open spaces.

In addition to the effect of the two integrated layers on thermal comfort level of the city, the void and volume layers integration has tremendous impact on the transportation layer. The density, defined in terms of the ratio between volume 
and void, plays a key role on the transportation layer and travel time such as the transportation differences between diffuse cities and dense cities, for instances.

Moreover, integration of the void layer with function layer, another layer which is under discussion in this paper, creates the diversity of the city. The diversity distribution of the different functions, in public open spaces as well as indoor spaces such as urban piazzas and shops coincides with probability of different urban activity and occurrence of the public to encounter and mingle for social and economical events. The convoluted integration of the three layers carries the complexity feature of the city. To reiterate, the porosity alongside the diversity, that being the vertical adaptation between the three principle layers for volume, void and function, forms the complexity of the urban system. In the horizontal adaptation, by either transforming a single element or adding a new building could optimize the natural ventilation and solar exposure. On the other hand, in the vertical adaptation, the volume and void layers could optimise the transportation network by providing the best optimal density.

\subsubsection{Functional layer}

Transformation of the functional layer, alike the other layers, modifies and hybridizes with its elements and the other subsystems. Modification via horizontal adaptation of the functional layer's element has a great effect on the other layers and subsystems, economic and social layer for instance, which are not under the investigation of the paper; however, the role of functional layer becomes apparent following hybridization with the other layers. This vertical adaptation, which is under investigation in this paper, is highly influenced by the total energy balance of the city.

Juxtaposition of different functions integrated into the volume layer creates the proximity of the spaces, fig.1. The result of functional layer and the volume layer integration, proximity, deeply affects the transportation layer. In simple words, the functional layer has to be transformed in such a way to improve the other layers' efficiency, particularly the transportation layer efficiency by reducing the travel demands as well as the travel time.

The adaptive modification of the layers and symbiotic action between them create a new hybrid form of the system, which could be observed in mixed-used urban patterns. Refers to Zanni [4], from a typological point of view, the design of hybridization [integration] works against to the reproduction of the monofunctional clusters; it works against the gated communities, against the agglomeration of shopping malls, against the proliferation of low rise residential areas.

Apart from integration of the functional layer with the transportation layer, accessibility, the relationship between form, volume layer's feature, and functional layer opens another investigation discussion and it lies beyond the scope of the presented paper. However, a lot of research has investigated the relationship between volume and function layers utterly; hence this paper does not investigate how the function could be optimized by volume layer and its form. 


\subsubsection{Transportation layer}

Transportation effects on greenhouse emission and fossil fuel energy consumption is conspicuous and crystal clear. Due to the major role of the transportation layer impact on total energy consumption of the city, even minor optimization of this layer could lead to significant impact on the total energy consumed by the city. To carry the simulative optimization methodology forward, one should focus more on horizontal and vertical adaptation of the transportation layer. Alike the other layers, the transportation layer transforms through its elements adaptation, horizontally as well as vertically. However, the significance of the transportation role, on urban morphology and urban energy balance, emerges from the vertical adaptation with the other principle layers, particularly the functional layer and volume layer. The transportation layer transforms in a way to increase the accessibility between functional layer elements. As discussed before, integration between volume layer and functional layer brings proximity to the urban morphology; proximity alongside the accessibility create the compactness feature of the city, with significant influence on total urban energy balance; the compactness is derived from the vertical collaboration of three principle layers, namely volume, function and transportation. The integration of the two key factors, proximity and accessibility, to form the compactness feature has been experienced in the mixed-used dense urban fabrics in many cities around Europe for instance.

In addition to the importance of the integration between the transportation layer and the other layers, the transportation layer, as component of a CAS, could act as a catalyst for the other layers transformation, and vice versa. The density, the volume layer characteristic feature, strongly influences the transportation layer transformation. To reiterate, every modification in urban density and the volume layer requests further adaptation of transportation layer and in the other way also, density could be altered to make the transportation layer more efficient and the functional layer more accessible.

\subsubsection{Additional layers}

As it is mentioned in the simulative analysis, selecting the proper involved principle layers among the numerous involved layers, which have the direct influence on the urban morphology and energy consumption of the city, is the key factor of the simulation; however there are more layers which play a role in this scenario, though not as significant as the aforementioned principle ones. These secondary layers are in the second level of importance with respect to the four mentioned principle layers, volume, void, function and transportation. One can involve the additional layers if more complex analysis and more accurate result want to be obtained.

One of these secondary layers is the green layer. The green layer has a considerable impact, though not as well as the principal layers, on urban morphology as well as urban energy consumption balance. This layer could address the harsh weather conditions in the regions with rough climate. Green layer hybridization with urban volumes, mitigate the cold wind exposure to the 
individual buildings, as well as over solar heating prevention during the summer time; moreover, this layer integration with urban voids moderates the negative heat island effect of the neighbourhoods. Additionally, symbiotic relationship the between green layer and transportation layer, thanks the 'proximity' derived from volume layer and functional layer integration, increases the willingness of using reduced energy transportation means such as bicycles. This increase is due to the new form of space, which has been created by the green layer integration with the void and volume layer. In the other words, the green layer not only has an impact on lowering the energy consumption of the city, but also could alter the urban space to a new form of space. According to Zanni [4], the urban hybrid [green layer hybridization and volume layer] is the fusion between "nature" and "artifice" in all its forms and its cultural dimensions. This is characteristic of each piece of architecture; the juxtaposition of artifice and nature incorporates parts of opposing realms, ultimately building a new hybrid organism.

\subsection{Transformation}

To forward the theoretical preceding parts, this section is a succinct depiction of scale of transformation intervention in an existing context, based on integrated design, which is the main premise of the paper. The intervention, which could be either modification of existing members or introduction of new elements, is carried out through horizontal and vertical adaptation. Eventually, the series of interventions, the continuous modification of the city elements, is proceeded with the urban transformation, over time, fig. 2.

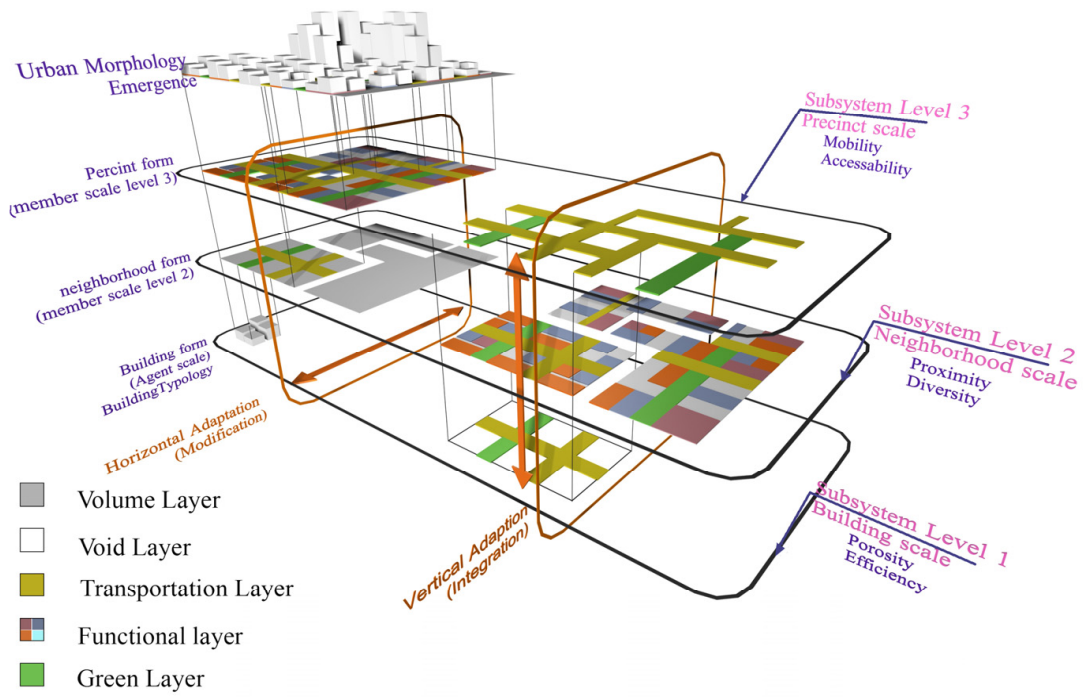

Figure 2: Intervention scales, horizontal and vertical adaptation at different scales, superimposing of the three different scales of transformation emerge the urban morphology. 
The scale of interventions, according to this complex system transformation methodology, is neither confined to a defined scale, nor to certain subsystems; however, the scale of classification, as presented in the paper, is just a way to explicate the transformation process, based on the paper hypothesis. The intervention is based on a holistic, multi-layer and multi-scale approach.

The intervention approach here is illustrated in three different scales, small scale, mid-scale and the large scale, as shown in fig. 2. In fact the complexity approach embraces the wide range of issues; the size and the scale of these approaches are defined by the intervention objectives. Since the main objective of this paper is urban transformation, the small scale intervention is a building scale, whilst the precinct level has been considered as the large one. Eventually, the urban morphology emerges by superimposition of the different scale interventions.

It is quite conspicuous that the intervention scale could be different than the mentioned scales, dependent upon the intervention size and its objectives. The large scale could be a territorial intervention, instead of precinct scale as represented in this paper, while the small scale could be a regional project, for instance.

The main aim of urban intervention's scale classification is solely an explanatory method. The intervention scale approach is hierarchy independent; thus, one should approach the urban design problem without sequential scale priorities.

\section{Conclusion}

The city, as a complex adaptive system, is not solely a mere aggregation of disconnected energy consumers and the total energy consumption of the city is different than the sum of all the building's consumption. This considerable gap between total energy consumption of the city and sum of all consumers is concealed in the urban morphology and urban form of the city.

Accordingly, the significance of urban morphological transformation toward a sustainable form has come into its own, due to its ability to tackle both environmental as well as urban growth problems simultaneously.

The investigation of urban transformation to the urban sustainable form has arisen the researchers' main concern; however, due to simplifying the problem by neglecting its complexity, the researchers have concluded with irreconcilable results. The complex system, the city, must be studied with inclusion of its complexity if more accurate results are to be obtained; hence, the investigations based on simulation are far preferable to simplification of the complex system.

The simulation methodology, based on complex adaptive system analysis, explicates a way to propel the urban transformation to the sustainable urban form. One can utilize this integrative methodology, based on modification and integration of existing elements, to propel the gradual transformation process into an appropriate direction. The main simulation lays on the premise of symbiotic relationship between members and subsystems, inside the complex adaptive system. According to this methodology, the energy efficiency of every 
element has to be optimised by its form; additionally, this element has to be designed in such a way to improve the other elements energy performances as well. This collaborative transformation method could be implemented practically, thanks to the horizontal adaptation via modification of the elements, and vertical adaptation via integration of subsystems, which are the two main complex adaptive system features.

The main four subsystems, involved in energy consumption of the city and urban morphology, which are used in the simulation, are the volume layer, void layer, functional layer and transportation layer. These layers, with the adaptive modification of their elements in one hand and the collaborative integration with the other layers in the other, transform the city to the sustainable form. Planners and designers could implement the integrated methodology by approaching the design and urban transforming task in the different scales. Ultimately the urban morphology emerges through superimposition of these three scales.

This research, the theory of the simulative integration methodology, is proceeded with the practical implications in different case studies. The case studies illustrate how the sustainable urban morphology emerges, through the modification and integration of the existing elements of the city. According to that, the case studies will be presented in the next phase of the research.

\section{References}

[1] Tadi, M., Is just a question of form? Energy Environment, .... THE HYBRID_LINK, 1, pp. 1-11, 2011.

[2] Mahaffy, M., Cowan, S. \& Urge-Vorsatz, D., The Factor of Urban Morphology in Greenhouse Gas Emission. IARU Scientific Congress: Copenhagen, pp. 1-12, 2009.

[3] Vahabzadeh Manesh, S. \& Tadi, M., Sustainable urban morphology emergence via Complex Adaptive System analysis. Procedia Engineering, 21, pp. 89-97, 2011.

[4] Zanni, F., Urban Hybridization. in press, pp. n/a, 2012.

[5] Brownlee, J., Complex Adaptive Systems. CIS Technical Report 070302A, pp. 1-6, 2007.

[6] Wootton, B. Cities as Complex Adaptive Systems. http://www.benjaminwootton.co.uk/

[7] Ratti, C., Baker, N. \& Steemers, K., Energy consumption and urban texture. Energy and Buildings, 37(7), pp. 762-776, 2005.

[8] Agencia d'Ecologia Urbana de Barcelona. Indicators that Characterise the Compact, Complex, Efficent and Stable Mediterranean City Model. Barcelona, www.ecourban.net

[9] Salat, S., The Art of Medieval Sustainable Design: The case of Toledo. SB10mad: Sustainable Building Conference, pp. 1-14, 2010. 